\title{
CP violation in QCD
}

\author{
Michael Creutz ${ }^{*+}$ \\ Brookhaven National Laboratory \\ E-mail: mikealatticeguy. net
}

Among the parameters of QCD is one that results in CP violation when non-vanishing. This is closely related to possible quark mass terms. It is conventionally interpreted in terms of gauge field topology or alternatively in terms of phases in the quark masses. There is no experimental evidence for this parameter having a non-zero value, a puzzle for theories involving unification.

XIII Quark Confinement and the Hadron Spectrum - Confinement2018

31 July - 6 August 2018

Maynooth University, Ireland

\footnotetext{
* Speaker.

${ }^{\dagger}$ Notice: This manuscript has been co-authored by employees of Brookhaven Science Associates, LLC under Contract No. DE-SC0012704 with the U.S. Department of Energy. The publisher by accepting the manuscript for publication acknowledges that the United States Government retains a non-exclusive, paid-up, irrevocable, world-wide license to publish or reproduce the published form of this manuscript, or allow others to do so, for United States Government purposes.
} 


\section{Introduction}

QCD depends on a remarkably small number of parameters. In conventional discussions these include the strong coupling constant $\alpha_{s}$, the quark masses $m_{i}$, and the topological parameter $\Theta$. But unlike with QED, the theory of electrons and photons, the connection with physical observables is rather non-trivial. In electrodynamics, both the electric charge and the electron mass are directly observable.

In QCD, because of asymptotic freedom [2, 3, 4] and dimensional transmutation[5], the strong coupling constant is tied to the overall scale. That in turn is connected with the particle masses. A natural scale to use is the mass of the proton; once that is determined the strong coupling constant is no longer an adjustable parameter. The quark masses are most directly tied to the pseudo-scalar spectrum. They can be adjusted to give the correct masses to the pions, kaons, etc.

The parameter $\Theta$ is perhaps the most subtle. When non-zero, this gives rise to CP violation. It is thus natural to adjust it to give the correct neutron electric dipole moment. Since such has not been seen, we know that $\Theta$ is either zero or very small. This is the strong CP problem.

At the heart of these issues is the confinement phenomenon. The underlying quarks are not free particles. The connection to the scattering of physical particles is subtle, and ambiguities, such as the so called renormalons, can arise. These ambiguities are closely related to defining $\Theta$, a non-trivial task since typical fields in the path integral are non-differentiable. The present talk summarizes some of these issues. Many of these topics are covered in considerably more detail in Ref. [1].

\section{Quark masses and $\Theta$}

Looking back a previous editions of this meeting, I see that many of my contributions were closely related to this subject. This is particularly true of my presentation at the 1996 edition in Como [6]. There I started by considering a naive variable change on a quark field

$$
\psi \longrightarrow e^{i \gamma_{5} \Theta} \psi
$$

This will modify the usual mass term

$$
\bar{\psi} \psi \longrightarrow \cos (\Theta) \bar{\psi} \psi+i \sin (\Theta) \bar{\psi} \gamma_{5} \psi
$$

This suggests that it might interesting to study a generalized mass term

$$
m \bar{\psi} \psi \rightarrow m_{1} \bar{\psi} \psi+i m_{5} \bar{\psi} \gamma_{5} \psi
$$

and explore how QCD depends on the two parameters $m_{1}$ and $m_{5}$. Were the above change of variables valid, one might expect physics to only depend on the combination $\sqrt{m_{1}^{2}+m_{5}^{2}}$. We will shortly see that this is false.

Our tool is an effective chiral Lagrangian. Working with two flavors, consider the usual "Mexican hat" or "wine bottle bottom" potential

$$
V=\left(\sigma^{2}+\vec{\pi}^{2}-v^{2}\right)^{2}-m_{1} \sigma
$$




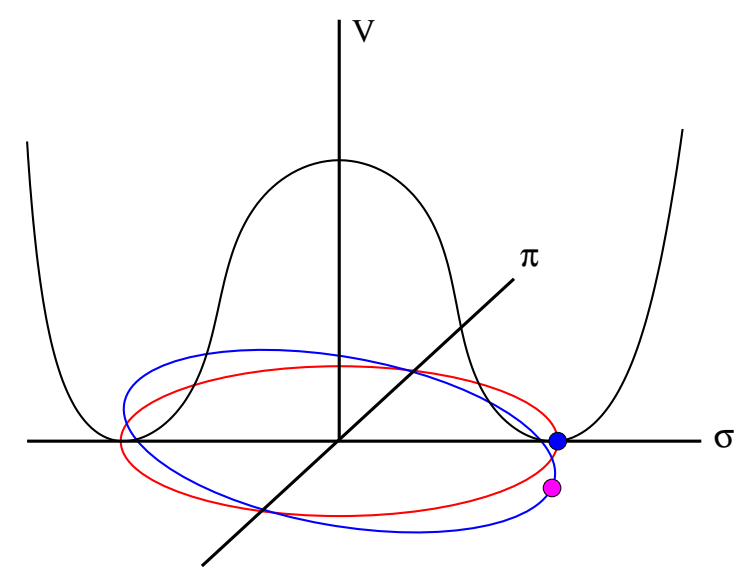

Figure 1: The starting effective potential. The mass term results in the minimum being in the positive $\sigma$ direction.

Here the mass term $m_{1} \bar{\psi} \psi \longrightarrow m_{1} \sigma$ tilts the sombrero, thereby putting the lowest energy state at positive sigma and giving the pion a small mass $M_{\pi}^{2} \propto m_{1}$. This is sketched in Fig. (1).

So in this picture, what does $m_{5}$ do? Indeed, a term like

$$
\mathrm{im}_{5} \bar{\psi} \gamma_{5} \psi \longrightarrow m_{5} \eta
$$

does not appear in the above effective potential. The effect of $m_{5}$ is of higher order in the chiral theory. The first thing $m_{5}$ does is to induce an expectation value for the eta field

$$
\langle\eta\rangle \propto m_{5} / M_{\eta}^{2} .
$$

To proceed, note that the flavored chiral rotation $\psi \rightarrow e^{i \tau_{3} \gamma_{5} \Theta}$ mixes the eta field $i \bar{\psi} \gamma_{5} \psi \sim \eta$ with the isovector scalar field $\bar{\psi} \vec{\tau}_{3} \psi \sim a_{03}$. The combination $\left(\eta, \vec{a}_{0}\right)$ represents a chiral pair in direct analogue with the original combination $(\sigma, \vec{\pi})$. Flavored chiral symmetry is consistent with a coupling between these fields of form

$$
\sim\left(\left(\begin{array}{cc}
\sigma & \vec{\pi}
\end{array}\right) \cdot\left(\begin{array}{c}
\eta \\
\vec{a}_{0}
\end{array}\right)\right)^{2}
$$

Here the square appears to preserve parity symmetry.

With an expectation for eta this gives an effective term

$$
\left(\sigma \eta+\vec{\pi} \cdot \vec{a}_{0}\right)^{2} \rightarrow\langle\eta\rangle^{2} \sigma^{2} .
$$

Including this in our original potential, $m_{5}$ induces a distortion proportional to the sigma field squared

$$
V \rightarrow V-\alpha m_{5}^{2} \sigma^{2} .
$$

where $\alpha$ is an undetermined constant. The sign of this term is related to pi-eta mixing. This gives rise to a quadratic warping of the effective potential, as sketched in Fig. 2.

From this argument, we see that $m_{5}$ also gives the pions a mass

$$
M_{\pi}^{2} \propto m_{5}^{2} .
$$




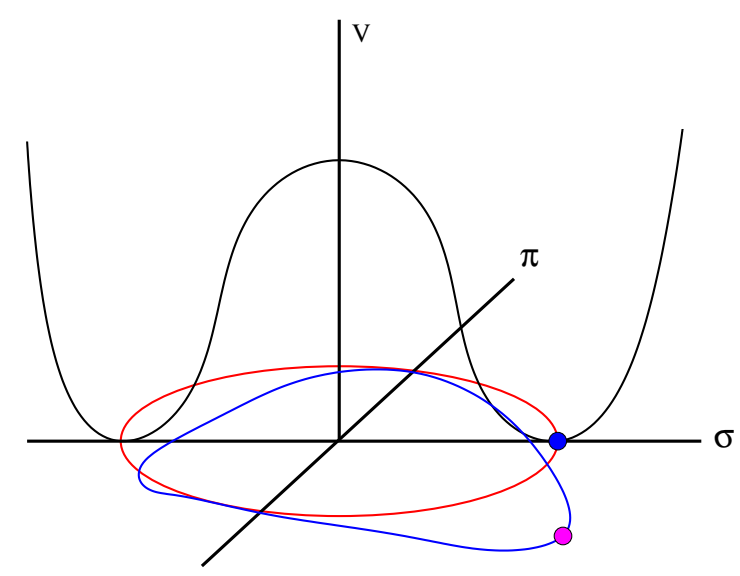

Figure 2: Including the $m_{5}$ term in the theory generates a quadratic warping of the effective potential.

It is crucial to note that unlike the original mass $m_{1}$ this is quadratic and not linear in $m_{5}$. Also note that this term induces a barrier between $\sigma>0$ and $\sigma<0$. If we look at the structure of the theory as a function of the two parameters $m_{1}$ and $m_{5}$, whenever $m_{5}$ is non-vanishing a first order transition appears at $m_{1}=0$. This is sketched in Fig. 3 .

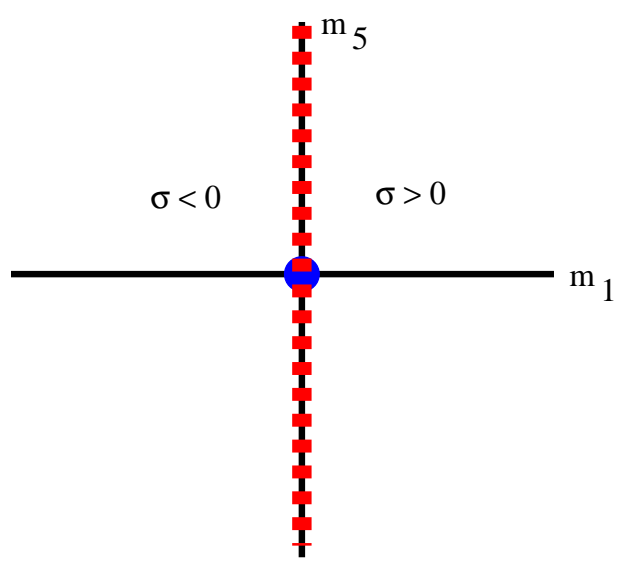

Figure 3: The phase structure as a function of $m_{1}$ and $m_{5}$ exhibits a first order transition along the $m_{5}$ axis. The transition is denoted by the dashed line and occurs when the conventional parameter $\Theta$ takes the value pi.

The transition occurs when the conventional parameter $\Theta$ is $\pi$. The mapping between $\Theta$ and the mass parameters is given by

$$
\frac{m_{5}}{m_{1}}=\tan (\Theta / 2)
$$

The crucial conclusion is that although Eq. (2.1) may look like a harmless change of variables, physics does not only depend on the combination $\sqrt{m_{1}^{2}+m_{5}^{2}}$. The rotation in Eq. (2.2) is "anomalous," and physics depends non-trivially on $\Theta$. The important point of this section is that $m_{1}$ and $m_{5}$ are physically independent parameters. 


\section{Why is $\psi \longrightarrow e^{i \gamma_{5} \Theta} \psi$ not a symmetry?}

The fact that gamma five rotations are anomalous has been known for some time [7, 8, 9]. However a deep connection between $\Theta$ and the fermionic measure in the path integral was later elucidated in the work of Fugikawa [10]. When a gauge field configuration has non-trivial topology, the Dirac operator has chiral zero modes. The index theorem relates the number of these modes to the topological index of the gauge field

$$
n_{+}-n_{-}=v
$$

Here $v$ is the gauge field winding number, and $n_{+}\left(n_{-}\right)$counts the number of left (right) zero modes. Now if we define the trace of $\gamma_{5}$ via a sum over the modes of the Dirac operator, we find that this trace need not vanish

$$
\operatorname{Tr} \gamma_{5} \equiv \sum_{i}\left\langle\psi_{i}\left|\gamma_{5} \cdot\right| \psi_{i}\right\rangle=v
$$

On configurations carrying topology, the chiral change of variables will introduce a phase in the fermionic measure of the path integral

$$
d \psi \rightarrow e^{i v \Theta} d \psi
$$

Thus this change of variables is equivalent to inserting a factor of $e^{i v \Theta}$ into the path integral

$$
Z=\int(d A)(d \psi)(d \bar{\psi}) e^{-\beta S} \longrightarrow \int(d A)(d \psi)(d \bar{\psi}) e^{i v \Theta} e^{-\beta S} .
$$

This represents a physically different theory. With $\Theta$ present, the theory is CP violating since the $m_{5}$ term is. This emphasizes yet further that $m_{1}$ and $m_{5}$ are inequivalent parameters that have nothing to do with each other.

A natural question at this point is whether there is an independent $m_{5}$ for each flavor? The answer is no because flavored chiral symmetries remain valid. A change of variables such as

$$
\psi \longrightarrow e^{i \gamma_{5} \lambda_{\alpha} \Theta} \psi
$$

is a valid symmetry when $\lambda_{\alpha}$ is one of the trace less generators of the flavor group. Rotations of this form allow us to move the chiral phase between different flavors. It is perhaps amusing to consider rotating any non-trivial $\Theta$ into the top quark. In this sense, the top quark does not fully decouple from low energy physics.

\section{The strong CP problem}

The parameter $m_{5}$ is inherently $\mathrm{CP}$ violating. But the experimental bounds on such a symmetry breaking are extremely small, of order $10^{-10}$ for the ratio $\frac{m_{5}}{m_{1}}$. Why should this be so?

This is actually only a problem for theories that unify the strong with the electro-weak interactions. We know experimentally that the weak interactions do violate $\mathrm{CP}$. Thus on reducing energies from the weak scale, it would be "natural" for a residue of this breaking to to survive in the low energy strong interactions. On the other hand, if the strong interactions are never unified with the others, then the symmetries of the theory make $\Theta=0$ stable under renormalization. 
One possible solution to this conundrum involves introducing a new particle called the axion. It is arranged to make the parameter $\Theta$ into a dynamical field with the ground state of this field at $\Theta=0$. Effectively this corresponds to adding something like $\left(\partial_{\mu} \Theta\right)^{2}$ to the action. The coefficient of this is arbitrary, and thus the coupling of the axion particle to the strong interactions can be made arbitrarily small.

While potentially viable, this procedure seems rather ad hoc and involves introducing a new particle. One might also worry that on descending from the unification scale a linear term in the $\Theta$ field might survive to give an expectation value to the $\mathrm{CP}$ violating effect.

\section{The up-quark mass}

It has been occasionally suggested that a vanishing up-quark mass would solve the strong $\mathrm{CP}$ problem. The argument is that flavored rotations can put all phases in the up-quark mass, and if the up-quark mass vanishes, it cannot have a phase. The shortcoming of this naive argument is that the up-quark mass involves the two parameters $m_{1}$ and $m_{5}$, and these parameters are independent. The strong CP problem is why is $m_{5}$ small, and has nothing to do with the parameter $m_{1}$.

Nevertheless, the possibility of a vanishing up-quark mass has received considerable attention over the years, and thus it is interesting to explore what this means. To begin with, we know that the pion masses are non-zero, and thus both $m_{u}$ cannot $m_{d}$ vanish. So to proceed we introduce an up-down mass difference term to the theory

$$
m \bar{\psi} \psi \rightarrow m_{1} \bar{\psi} \psi+m_{2} \bar{\psi} \tau_{3} \psi
$$

The term $m_{2} \bar{\psi} \tau_{3} \psi \sim a_{03}$ transforms as an isovector scalar. This, just as the $m_{5}$ term, does not appear in the starting effective potential of Eq. (2.4). To study its effect, we parallel the earlier discussion on $m_{5}$ and note that $m_{2}$ will give $a_{03}$ an expectation value

$$
\left\langle a_{03}\right\rangle \propto m_{2} / M_{a_{0}}^{2}
$$

This will enter the same effective term as in Eq. (2.7) and warp the effective potential. But now the distortion is downward in the $\pi_{3}$ direction,

$$
V \rightarrow V-\alpha m_{2}^{2} \pi_{3}^{2}
$$

If we do not include an overall tilt in the $\sigma$ direction, the $\pi_{3}$ field will gain an expectation value! This represents the so called the CP violating "Dashen phase" [11]. This situation is sketched in Fig. 4.

To understand this phase better, consider fixing the down quark mass to some positive value and vary the up-quark mass. As the up-quark becomes lighter, the pions will decrease in mass, as sketched in Fig. 5. Note that the pions remain massive as the up quark goes to zero. At that point the mass gap of the theory remains, and no singularity occurs. As we continue the up-quark mass into the negative regime, the pions continue to become lighter, with the breaking of isospin making the neutral pion lighter than the charged ones. But if we make the up-quark mass sufficiently negative, the neutral pion mass can vanish. Beyond that point $\pi_{3}$ gains its expectation value, and 


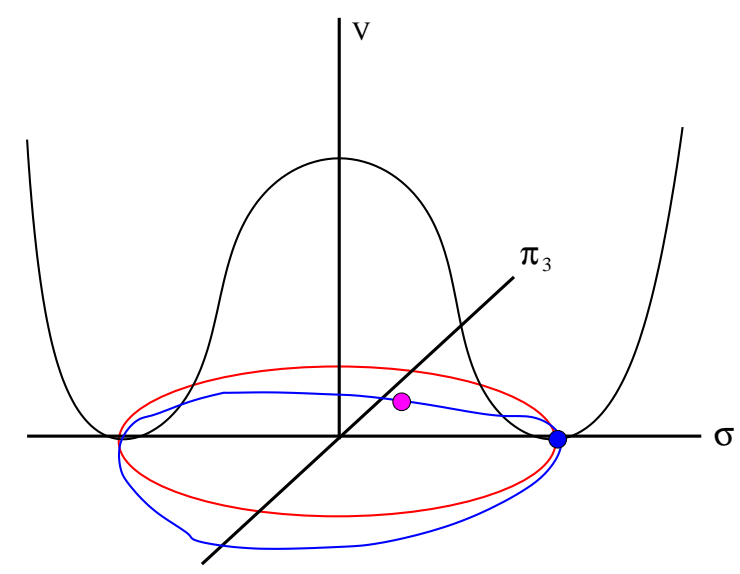

Figure 4: The presence of a quark mass difference distorts the effective potential downward in the $\pi_{3}$ direction.

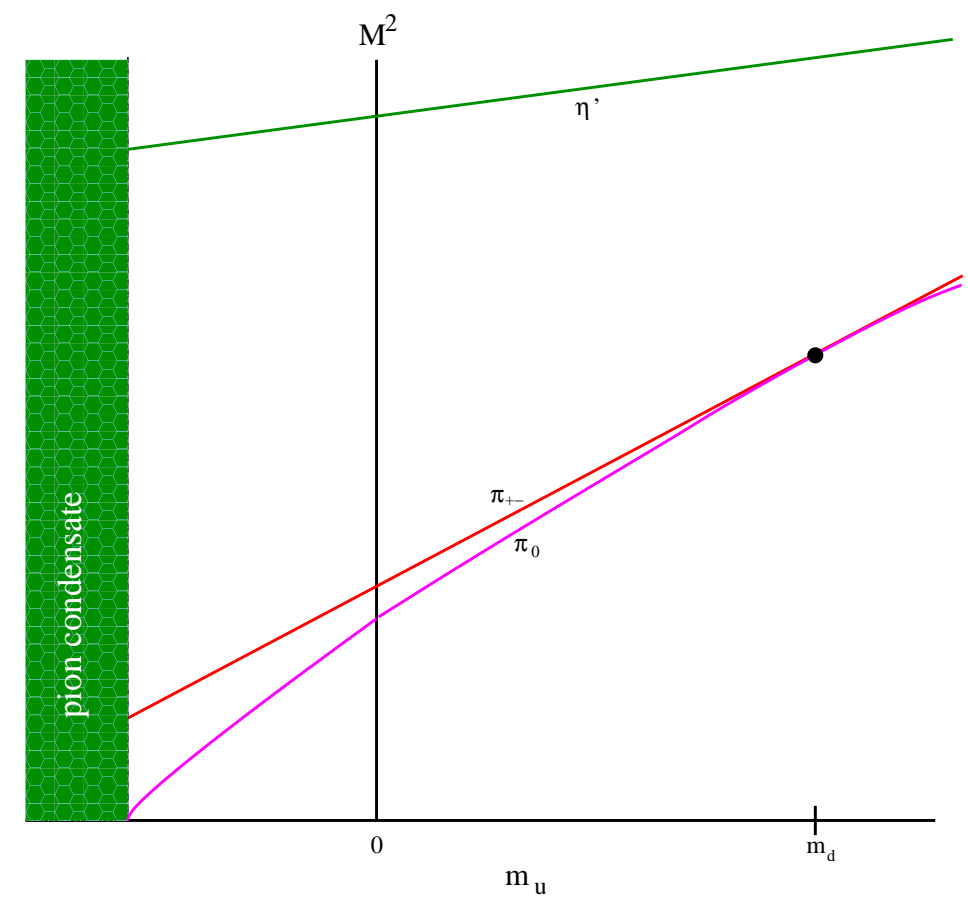

Figure 5: As the up-quark mass is reduced below that of the down quark, isospin breaking separates the charged and neutral pion. As the up-quark goes through zero mass, no singularity is expected. If the upquark mass becomes sufficiently negative, the neutral pion can condense and we enter the $\mathrm{CP}$ violating Dashen phase. 
we are in the Dashen phase. Note that in this regime the product of the quark masses is negative and we are formally at $\Theta=\pi$.

We now have at a rather simple picture for the qualitative behavior of two flavor QCD as a function of the conventional parameters $\alpha_{s} m_{u} m_{d} \Theta$. These map in a non-linear way into the overall scale, the tilt of the effective potential, and a possible quadratic warping. However in general the tilt and warp need not be in the same direction, and the fourth parameter represents a possible angle between them, as sketched in Fig. 6 .

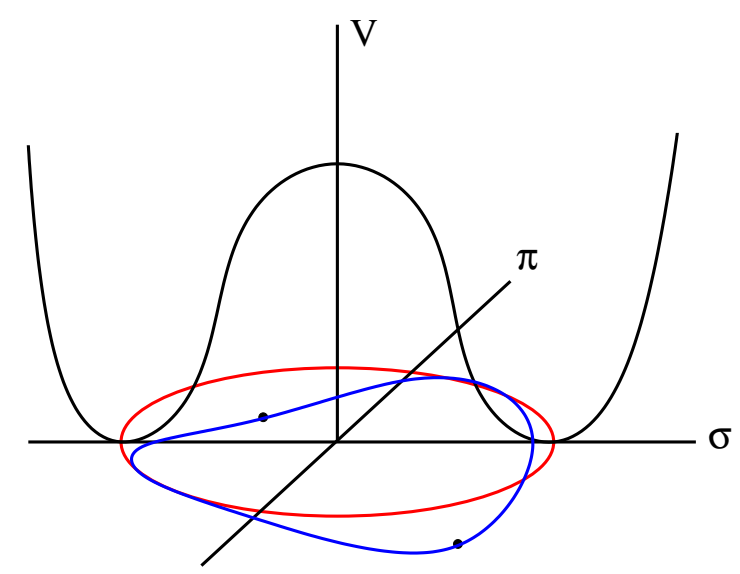

Figure 6: The four parameters of two flavor QCD map onto the effective chiral Lagrangian as the overall scale, the tilt, the warp, and the angle between the tilt and warp.

Extending these arguments, we can obtain the full two flavor phase diagram as a function of the parameters $m_{1}, m_{2}, m_{3}$ [12] as sketched in Fig. 7. The plane at $m_{1}=0$ extends the first order transition from Fig. 3. In addition, there is another first order transition extending into the $m_{5}=0$ plane; the order parameter here is the sign of the non-vanishing expectation for the $\pi_{3}$ field.

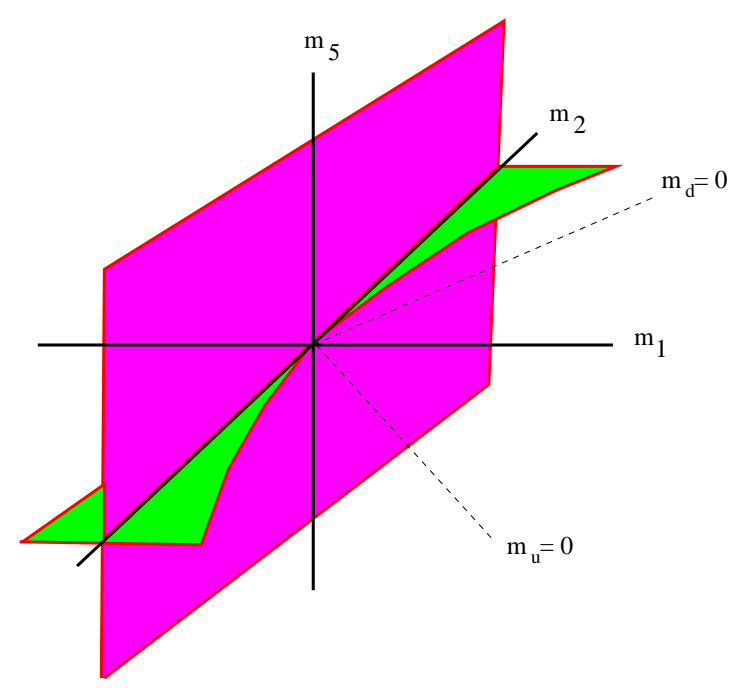

Figure 7: The full phase diagram for two flavor QCD as a function of $m_{1}, m_{2}, m_{3}$. 


\section{Symmetries in the masses}

This diagram has a variety of symmetries. First, it is invariant under changing the sign of the parameter $m_{5}$. This is associated with CP and will protect $m_{5}$ from any additive renormalization. What about the other mass parameters? To proceed, concentrate on $m_{5}=0$ plane, sketched in Fig. 8. At the edge of the Dashen phase is a second order transition where the neutral pion becomes mass-less. The order parameter for the transition is the expectation value for the neutral pion field, $\left\langle\pi_{0}\right\rangle$.

The next invariance to note is under exchanging the up and down quark masses. This is isospin, and protects the quark mass difference $m_{2}$ from additive renormalization. Then there is a symmetry under $m_{u} \leftrightarrow-m_{d}$. This represents isospin at $\Theta=\pi$ and protects $m_{1}$ from additive renormalization. Another symmetry, not really independent of the above, is under flipping the signs of both quark masses. This can be implemented by a flavored chiral rotation

$$
\psi \rightarrow e^{i \pi \tau_{3} \gamma_{5}} \psi
$$

which is not anomalous.

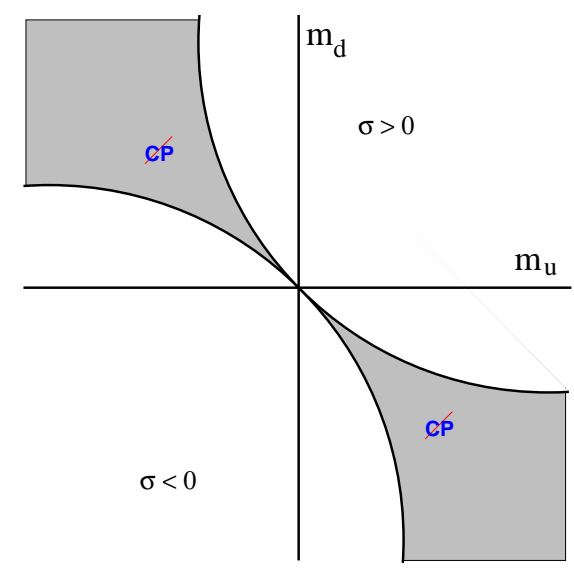

Figure 8: The phase diagram for two flavor QCD at $m_{5}=0$ as a function of the up and down quark masses.

A crucial observation is that this diagram is not symmetric under $m_{u} \leftrightarrow-m_{u}$. The concept of a vanishing up-quark mass is not protected by any symmetry! While symmetries protect the three parameters $m_{1}, m_{2}, m_{3}$ individually, these quantities in general can have independent renormalizations. One might try to define

$$
\text { " } m_{u} "=\frac{m_{1}+m_{2}}{2}+i m_{5}
$$

however this combines independent parameters and is an artificial construct.

So this leaves us with a conundrum. Can any experiment tell if the up quark mass vanishes? If not, is $m_{u}=0$ a well defined concept? The issues here are all non-perturbative, so relating the up-quark mass to a perturbative definition cannot answer this. Non-perturbative issues require a regulator such as the lattice. And at least naively the lattice can answer the question. One should adjust the lattice parameters until the physical hadron spectrum comes out right. Then one can read off the input lattice quark masses and see if $m_{u}=0$. 
There is a complication to this process. As shown by 't Hooft some time ago [13], a nonvanishing down quark mass can induce an effective mass for the up-quark. Both the combinations $i \bar{u} \gamma_{5} u$ and $i \bar{d} \gamma_{5} d$ couple to the pion. This means that through the pion field, a left handed upquark can turn into a right handed one by an amount proportional to the down quark mass. This is sketched in Fig. 9. Because of this effect, ratios of quark masses are not renormalization group invariant when non-perturbative effects are taken into account.

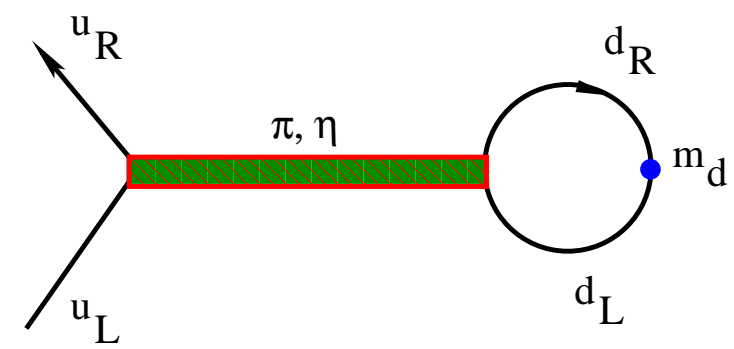

Figure 9: A non-vanishing down quark mass can, through the pion field, convert a left handed up quark into a right handed one.

Can we use the topology of the gauge fields to get a handle on this issue? The fermion determinant suppresses non-trivial topology when a quark mass vanishes. The concept of a vanishing quark mass is equivalent to the vanishing of the average gauge field topology. So this leaves us with the question of how to define lattice topology. This is also rather non-trivial. As is well known, typical configurations in the path integral involve non-differentiable fields. Indeed, the space of lattice fields is simply connected in most formulations. Topology is lost at the outset since small instantons can "fall through the lattice." Many studies over the years $[14,15]$ have attempted to get around this by some sort of cooling process to remove short distance fluctuations from the gauge fields. In this way the action is observed to settle into multiple well defined instantons. The problem is that while this procedure is often stable, it is not unique. The net winding can depend on the details of the cooling algorithm [16]. Additional questions are what action should we use to cool, and how long should we cool.

Can we use the index theorem to resolve this? Topology is associated with zero modes of the Dirac operator. So we might try to count the small real eigenvalues of the Wilson fermion operator. The issue here is that at finite cutoff these modes are not exact zeros. How should we define "small" for the eigenvalues? The result will depend on the density of real eigenvalues in the first Wilson circle, which in general need not vanish. One might instead count zero modes of the overlap operator [17], which do occur at the origin. The problem here is that the overlap operator is not unique, depending on a parameter often called the "domain wall height." This parameter is closely related to the Wilson operator and its eigenvalues.

Should we care if topology is ambiguous? This is an abstract theoretical construction and not something directly measured in laboratory experiments. One should concentrate on physical quantities, such as the mass of the eta prime. The famous Witten-Veneziano $[18,19]$ formula does relate topological susceptibility of the pure gauge theory to the eta prime mass, but this is only a result in the limit of a large number of colors. 


\section{Summary}

QCD depends on $N_{f}+1$ possible mass parameters, where $N_{f}$ is the number of fermion flavors. One of these is the CP violating parameter usually referred to as $\Theta$. The effects of $\Theta$ are not visible in perturbation theory. Indeed, theories with different values of this parameter have identical perturbative expansions.

Existing experiments have found no evidence for a non-vanishing value for $\Theta$. This is a puzzle for models of unification since $\mathrm{CP}$ violation is evident in weak interaction processes. One possible solution involves introducing an axion to make $\Theta$ into a dynamical field that then relaxes to zero. The possibility of $m_{u}=0$ is not a viable solution since it involves an unnatural fine tuning. Three unrelated parameters, $m_{1}, m_{2}$, and $m_{5}$, contribute to the up-quark mass. Only one of these is associated with $\mathrm{CP}$ violation. The other two have nothing to do with the puzzle.

\section{References}

[1] M. Creutz, "From Quarks to Pions : Chiral Symmetry and Confinement," doi:10.1142/10688

[2] H. David Politzer. Reliable Perturbative Results for Strong Interactions? Phys. Rev. Lett., 30:1346-1349, 1973.

[3] D.J. Gross and Frank Wilczek. Ultraviolet behavior of nonabelian gauge theories. Phys.Rev.Lett., 30:1343-1346, 1973.

[4] D.J. Gross and Frank Wilczek. Asymptotically free gauge theories. 1. Phys.Rev., D8:3633-3652, 1973.

[5] Sidney R. Coleman and Erick J. Weinberg. Radiative corrections as the origin of spontaneous symmetry breaking. Phys.Rev., D7:1888-1910, 1973.

[6] M. Creutz, "Quark masses, chiral symmetry, and the U(1) anomaly," hep-ph/9608216.

[7] Stephen L. Adler. Axial vector vertex in spinor electrodynamics. Phys.Rev., 177:2426-2438, 1969.

[8] Stephen L. Adler and William A. Bardeen. Absence of higher order corrections in the anomalous axial vector divergence equation. Phys.Rev., 182:1517-1536, 1969.

[9] J.S. Bell and R. Jackiw. A PCAC puzzle: pi0 to gamma gamma in the sigma model. Nuovo Cim., A60:47-61, 1969.

[10] Kazuo Fujikawa. Path integral measure for gauge invariant fermion theories. Phys.Rev.Lett., 42:1195, 1979.

[11] Roger F. Dashen. Some features of chiral symmetry breaking. Phys.Rev., D3:1879-1889, 1971.

[12] Michael Creutz. Quark mass dependence of two-flavor QCD. Phys. Rev., D83:016005, 2011.

[13] Gerard 't Hooft. Computation of the quantum effects due to a four-dimensional pseudoparticle. Phys.Rev., D14:3432-3450, 1976.

[14] M. Teper. Instantons in the quantized su(2) vacuum: A lattice Monte Carlo investigation. Phys.Lett., B162:357, 1985.

[15] Falk Bruckmann, Florian Gruber, Karl Jansen, Marina Marinkovic, Carsten Urbach, et al. Comparing topological charge definitions using topology fixing actions. Eur.Phys.J., A43:303-311, 2010. 
[16] Michael Creutz. Anomalies, gauge field topology, and the lattice. Annals Phys., 326:911-925, 2011.

[17] Herbert Neuberger. Exactly massless quarks on the lattice. Phys.Lett., B417:141-144, 1998.

[18] Edward Witten. Current algebra theorems for the $u(1)$ goldstone boson. Nucl.Phys., B156:269, 1979.

[19] G. Veneziano. $u(1)$ without instantons. Nucl.Phys., B159:213-224, 1979. 